\title{
Differential p16/INK4A cyclin-dependent kinase inhibitor expression correlates with chemotherapy efficacy in a cohort of 88 malignant pleural mesothelioma patients
}

\author{
C J Jennings ${ }^{1}$, B Murer ${ }^{2}$, A O'Grady ${ }^{3}$, L M Hearn ${ }^{1}$, B J Harvey ${ }^{1}$, E W Kay ${ }^{3}$ and W Thomas ${ }^{*} 1,4$ \\ ${ }^{1}$ Molecular Medicine Laboratories, Royal College of Surgeons in Ireland, Education and Research Centre, Beaumont Hospital, \\ Dublin 9, Ireland; ' ${ }^{2}$ Department of Anatomy and Histopathology, Dell' Angelo Hospital, Via Paccagnella 11, Mestre, 30174 VE, Italy; \\ ${ }^{3}$ Department of Histopathology, Royal College of Surgeons in Ireland, Education and Research Centre, Beaumont Hospital, \\ Dublin 9, Ireland and ${ }^{4}$ Perdana University - Royal College of Surgeons in Ireland School of Medicine, Jalan MAEPS Perdana, 43400 \\ Serdang, Selangor, Malaysia
}

\begin{abstract}
Background: Malignant pleural mesothelioma (MPM) is a rare and essentially incurable malignancy most often linked with occupational exposure to asbestos fibres. In common with other malignancies, the development and progression of MPM is associated with extensive dysregulation of cell cycle checkpoint proteins that modulate cell proliferation, apoptosis, DNA repair and senescence.
\end{abstract}

Methods: The expression of cyclin-dependent kinase inhibitor p16/INK4A was evaluated by immunohistochemistry using tumour
biopsy specimens from 88 MPM cases and a semi-quantitative score for p16/INK4A expression was obtained. Post-diagnosis
survival and the survival benefit of chemotherapeutic intervention was correlated with p16/INK4A expression.

Results: A low, intermediate and high score for p16/INK4A expression was observed for 45 (51.1\%), 28 (31.8\%) and 15 (17.1\%) of the MPM cases, respectively. Those cases with intermediate or high p16/INK4A tumour expression had a significantly better postdiagnosis survival than those cases whose tumours lost p16 expression (log-rank $P<0.001$ ). Those patients with sustained p16/ INK4A expression who received chemotherapy also had a better survival than those treated patients whose tumours had lost p16/ INK4A expression (log-rank $P<0.001$ ).

Conclusions: Sustained p16/INK4A expression predicts better post-diagnosis survival in MPM and also better survival following chemotherapeutic intervention.

Malignant pleural mesothelioma (MPM) is a rare malignancy with extremely poor prognosis that arises in the cells of the pleura, most often decades after occupational exposure to high concentrations of asbestos fibres (Sterman and Albelda, 2005). In spite of strict controls on asbestos use, the incidence of MPM is continuing to rise in many developed countries due to the potentially long latent period $(30+$ years $)$ of this malignancy following initial exposure to the carcinogen (Bianchi and Bianchi, 2007; Le Stang et al, 2010; Jennings et al, 2014). Histological subtype is the most accurate predictor of post-diagnosis survival, with patients diagnosed with an epithelioid type tumour typically having a 13.6 month median survival and patients with sarcomatoid-type cells having a 6.1 month median survival (Steele, 2005; Flores et al, 2007). Cell cycle dysregulation is a critical aspect of all malignancies and arises 
through the mutation, deletion, amplification or aberrant regulation of a set of critical genes (Sherr, 1996). Cell cycle progression is controlled by a group of proteins called cyclins that modulate the activity of a family of protein kinases termed cyclin-dependent kinases (CDKs), which in turn regulate the activity of specific transcription factors. The $\mathrm{CDKs}$ are also regulated by $\mathrm{CDK}$ inhibitors including p14/ARF, p16/INK4A, p15/INK4B, p21/Cip/ WAF1 and p27/Kip1, which show variable expression in different MPM tumours (Spugnini et al, 2007). In general the expression of $\mathrm{CDK}$ inhibitors is often lost in more aggressive tumours, correlating with worse survival.

The CDKN2A gene encodes the p14/ARF and p16/INK4A proteins. The activity of CDK-4/6 is inhibited by p16/INK4A expression, resulting in suppression of $\mathrm{Rb}$ hyperphosphorylation by $\mathrm{CDK}-4 / 6$ and $\mathrm{Rb}$ release from $\mathrm{E} 2 \mathrm{~F}$, a key transcriptional regulator at the G1/S checkpoint in the cell cycle (Burke et al, 2014). The loss of p16/INK4A expression, thus contributes to increased free E2F and uncontrolled growth by tumour cells. The p14 polypeptide is expressed from a related mRNA to p16 that has a different exon-1, and alternative splicing onto exon-2 resulting in translation in a different reading frame. The ubiquitin ligase MDM2 is regulated by p14, making p14 a potent tumour suppressor through its regulation of p53 ubiquitination and protein abundance. Homozygous deletion of CDKN2A is detected in a high proportion of MPM tumours (72\%), and in $85 \%$ of cultured MPM cell lines (Ichimura et al, 2005; Watkins et al, 2008). The loss of p16 expression is also detected in many other malignancies confirming its role as a tumour suppressor. In addition to deletion, the CDKN2A gene is also subject to hypermethylation leading to the silencing of expression without genetic loss (Watkins et al, 2008). The reconstitution of p16 expression in MPM cell lines results in cell cycle arrest and suppression of $\mathrm{Rb}$ phosphorylation, raising the question of whether there may be a potential genetic therapy approach to treat p16 deficient malignancies (Wong et al, 2002; Watkins et al, 2008). The strong association between the loss of p16 expression and MPM progression not only makes p16 abundance potentially useful in distinguishing MPM from non-malignant lung conditions, but also in predicting MPM post-diagnosis survival (Dacic et al, 2008).

In this present study we evaluated the expression of the p16/ INK4A CDK inhibitor in a well-characterised cohort of 88 confirmed MPM cases, and related p16/INK4A expression to post-diagnosis survival and to other patient and tumour characteristics associated with MPM survival.

\section{MATERIALS AND METHODS}

Patients and tissue samples. Eighty-eight cases of confirmed MPM and three control subjects were identified from the archival pathology files of the Pathology Unit of the Regional Hospital of Mestre-Venice, Italy. Each diagnosis of MPM was based on World Health Organization criteria, and in all instances, confirmed by clinical, immunohistochemical (IHC) and morphological evaluation. The tissue samples were taken from resected surgical specimens or following videothoracoscopy biopsy. The tissue samples were fixed in neutral formalin and embedded in paraffin. Permission for tissue to be used for research purposes was obtained according to local ethical procedures and following informed patient consent. Clinical data relating to each of the subjects was obtained with consent from primary patient records and coded before analysis by researchers.

Immunohistochemistry. Immunohistochemical parameters for the p16/INK4A-specific antibody were initially optimised using a breast carcinoma tissue micro-array. The IHC analysis for each antigen was performed using a Bond III Automated IHC Stainer
(Leica Microsystems, Wetzlar, Germany) on serial $4 \mu \mathrm{m}$ depth tissue sections from each of the embedded specimens. Slides were treated for $20 \mathrm{~min}$ with Leica BondMax Epitope Retrieval Solution 1 (ER1) to achieve post-sectioning antigen retrieval. The specific p16/INK4A primary antibody (SC-468 (C20), rabbit polyclonal antibody, 1 : 200; Santa Cruz Biotechnology, Santa Cruz, CA, USA) was applied for $20 \mathrm{~min}$ and revealed using the Leica Bond Polymer Refine detection kit (Leica Microsystems). The signal was enhanced using the Leica BondMax DAB enhancer kit (Leica Microsystems). Slides were counterstained with haematoxylin before mounting and microscopic visualisation.

Scoring system. The semi-quantitative determination of $\mathrm{p} 16 /$ INK4A expression was performed according to the criteria described by Allred et al (1993). The proportion of positive stained cells was scored as $0=$ no cells staining positive, $1=0-1 \%$ positive, $2=1-10 \%$ positive, $3=10-33 \%$ positive, $4=33-66 \%$ positive and $5=66-100 \%$ positive. In combination with the proportion score, an intensity score was attributed to each specimen on the basis of the average intensity of staining: $0=$ negative, $1=$ weak, $2=$ intermediate and $3=$ strong. The proportion score and the intensity score were added to obtain the total score, and is either 0 or a whole number between 2 and 8 . Allred scores of $\geqslant 6$ were interpreted as strong staining. Slides were independently evaluated and scored in a blind fashion by two independent observers. Any discrepancies arising in the scoring between the two observers were resolved by review of the specimens under a double-headed microscope and a consensus score was allocated.

Statistical analysis. Differences in post-diagnosis survival were calculated using Kaplan-Meier analysis and survival curves were generated using the TMA Foresight package (TMA Foresight 3.01, Premier Biosoft International, Palo Alto, CA, USA) as described previously (Jennings et al, 2012). $P$-values were calculated using a log-rank test (Harrington, 1982) and a $P$-value $<0.05$ was taken to be indicative of statistical significance between populations. Multivariate analysis of variance (MANOVA) was performed using the SPSS package (SPSS v21, IBM, Armonk, NY, USA). The expression of p16 was categorised by Allred score $(0-3$ or $>3)$, histological type was categorised as epithelioid, biphasic or sarcomatoid, age at diagnosis was categorised as $<55$ years or $\geqslant 55$ years, gender was categorised as male or female and therapy was categorised as with or without chemotherapy. Chemotherapeutic treatment and p16 expression were treated as independent variables. Gender, age at diagnosis and hitological type were treated as covariates.

Cell culture. The MPM cell lines used in this study were derived from tumours of epithelioid, biphasic and sarcomatoid subtypes. The epithelioid cell lines used were: REN (Smythe et al, 1994), MPP-89 (Orengo et al, 1999), MMP (Cacciotti et al, 2002), MMB (Cacciotti et al, 2002), MMCA (Cacciotti et al, 2002) and AccMeso-1 (Usami et al, 2006). The biphasic cell lines were: Y-Meso14 (Usami et al, 2006) and Msto-211H (Instituto Scientifico Tumori, Genoa, Italy). The sarcomatoid cell lines used were: H2052 (ATCC, Teddington, UK), Y-Meso-8D (Usami et al, 2006) and H2596 (ATCC). The Met-5A are a TERT-transformed normal mesothelial cell line (Ke et al, 1989). MCF-7 and T47D breast carcinoma cell lines were purchased from ATCC. All cell lines were routinely maintained in phenol red-free RPMI 1640 medium (Lonza, Slough, UK) supplemented with $10 \%$ foetal bovine serum at $37^{\circ} \mathrm{C}$ in a humidified atmosphere of $5 \% \mathrm{CO}_{2}$.

Quantitative PCR. Total RNA was extracted from sub-confluent cell monolayers using the RNeasy extraction kit (Qiagen, Crawley, UK) according to manufacturer's instructions. RNA was quantified and its purity was established using a NanoDrop 8000 spectrophotometer (Eppendorf, Cambridge, UK). Reverse transcription of 
isolated mRNA to synthesise cDNA was performed using the ImProm-II RT kit (Promega, Dublin, Ireland) according to manufacturer's instructions in a DNA-Dyad programmable heating block (Biorad, Dublin, Ireland). Real-time (RT) PCR was used to quantify the abundance of chosen mRNA species. Realtime PCR was performed using a 7500 Fast System and SYBR Green reagents (Applied Biosystems, Warrington, UK). The primer pairs specific for each of the mRNA species quantified in this study were derived from prior publications where their efficacy had been established experimentally (Kanellou et al, 2008; Li et al, 2009) (Supplementary Table 1). We confirmed the specificity of each of these primer pairs by BLAST (www.ncbi.nlm.nih.gov/BLAST/) database search, and we also confirmed the efficiency of each of the primer pairs under RT-PCR reaction conditions before analysis. The abundance of each of the mRNA species in the different cell lines was normalised against $18 \mathrm{~S}$ rRNA abundance and is expressed as relative abundance compared with T47D breast carcinoma cells. A relative abundance of $<0.001$ compared with T47D cells is treated as not detected.

Western blotting. Lysates were prepared from sub-confluent MPM cell lines grown on $10 \mathrm{~cm}$ diameter culture plates. Briefly, MPM cells were lysed in RIPA buffer $(1 \mathrm{ml})$ and the lysate was cleared by centrifugation ( $10000 \mathrm{~g}$ for $20 \mathrm{~min})$. Equal amounts of the lysates ( $50 \mu \mathrm{g}$ protein by Bradford Assay) were separated on a $12.5 \%$ resolving phase SDS-PAGE gel. The proteins were transferred to PVDF and probed with a p16-specific monoclonal antibody diluted 1:5000 (SC-56330, Santa Cruz Biotechnology) or a $\beta$-actin-specific monoclonal antibody diluted 1:5000 (A5316, Sigma-Aldrich, Arklow, Ireland). Primary antibodies were detected using rabbit anti-mouse horseradish peroxidase conjugate (SigmaAldrich) and visualised by enhanced chemiluminescence (GE Healthcare, Little Chalfont, UK). Exposed films were digitally recorded using Gene Snap software (Synoptics, Cambridge, UK).

Immunofluorescent imaging. The cell lines indicated were grown on $1 \mathrm{~cm}$ diameter coverslips for $24 \mathrm{~h}$, then washed in room temperature phosphate-buffered saline (PBS) and fixed in $4 \%$ paraformaldehyde in PBS. The cell membranes were disrupted in $0.2 \%$ Triton X-100 in TBS. Non-specific binding of antibodies and fluorescent conjugates were blocked by addition of $4 \%$ BSA and $2 \%$ fish skin gelatin (Sigma-Aldrich) in PBS. Bound p16 antibodies (SC-56330, 1:200) were detected using rabbit anti-mouse Alexafluor 488 conjugate (1:500; Invitrogen, Dun Laoghaire, Ireland). Tetramethylrhodamine isothiocyanate (TRITC) conjugated phalloidin was used to label the actin cytoskeleton. Cells were mounted in Vectashield (Vector Laboratories, Peterborough, UK) containing 4', 6-diamidino-2-phenylindole (DAPI) and examined using a LSM 510 Meta confocal microscope (Zeiss, Oberkochen, Germany). Excitation wavelengths for Alexafluor 488, TRITC and DAPI were 488,543 and $364 \mathrm{~nm}$, respectively. Images were captured at $\times 63$ magnification. Scans were performed at $0.9-\mu \mathrm{m}$ interval depths through the fixed cells and single or merged images are presented as XY single planes through the centre of the cells.

\section{RESULTS}

Patient cohort characteristics. The evaluation of p16/INK4A expression by the tumour cells in biopsy specimens from a welldefined cohort of MPM subjects with $>5$ years post-diagnosis follow-up was performed using IHC (Jennings et al, 2012). Paraffin-embedded tumour samples from 88 patients with an age range of 44-82 years, and three normal control subjects were subject to analysis. The cohort comprised 72 male and 16 female subjects (Table 1). The histological types were determined to be 71 (80.7\%) epithelioid, 9 (10.2\%) biphasic and 8 (9.1\%) sarcomatoid. Cisplatin-based chemotherapy had been given to a total of 64
Table 1. Summary of subject characteristics at diagnosis and p16/INK4A immunohistochemistry data $(n=88)$

\begin{tabular}{|c|c|c|c|}
\hline & Male $(n=72)$ & Female $(n=16)$ & All $(n=88)$ \\
\hline \multicolumn{4}{|c|}{ Histology type, n (\%) } \\
\hline Epithelioid & $56(77.8)$ & $15(93.7)$ & $71(80.7)$ \\
\hline Biphasic & $8(11.1)$ & $1(6.3)$ & 9 (10.2) \\
\hline Sarcomatoid & $8(11.1)$ & $0(0)$ & $8(9.1)$ \\
\hline \multicolumn{4}{|l|}{ Stages } \\
\hline T2 & $22(30.6)$ & $5(31.2)$ & $27(30.7)$ \\
\hline T3 & $40(55.6)$ & 5 (31.2) & $45(51.1)$ \\
\hline T4 & $10(13.8)$ & $6(37.5)$ & $16(18.2)$ \\
\hline \multicolumn{4}{|c|}{ Chemotherapy, n (\%) } \\
\hline Yes & $51(70.8)$ & $13(81.3)$ & $64(72.7)$ \\
\hline No & $21(29.2)$ & $3(18.7)$ & $24(27.3)$ \\
\hline \multicolumn{4}{|c|}{ p16 Expression } \\
\hline Negative/low & $39(54.2)$ & $6(37.5)$ & $45(51.1)$ \\
\hline Intermediate & $22(30.5)$ & $6(37.5)$ & $28(31.8)$ \\
\hline High & $11(15.3)$ & $4(25.0)$ & $15(17.1)$ \\
\hline
\end{tabular}

$(72.7 \%)$ of the subjects post diagnosis. Twenty of the patients received chemotherapy in combination with surgery and radiotherapy; 21 received chemotherapy with just surgery and three received chemotherapy in combination with just radiotherapy. Ten patients received chemotherapy alone (Supplementary Table 2). As described previously for this cohort, the median post-diagnosis survival time was 15 months for patients with epithelioid MPM, 10 months for subjects with biphasic mesothelioma and 6 months for subjects with sarcomatoid mesothelioma (Jennings et al, 2012). The median survival time for male subjects in this cohort was 10 months and 18 months for female subjects. These trends are consistent with other studies where epithelioid histological type and female gender are associated with better prognosis.

Expression of p16/INK4A in MPM tumours. The p16/INK4 CDK inhibitor is a critical regulator of G1/S transition in the cell cycle and its expression is lost or suppressed in many malignancies including MPM. We found in this present study that p16/INK4A was highly expressed in normal mesothelium and that there was variation in expression of p16/INK4A between the MPM tumour specimens (Figure 1). The Allred scoring system was applied to quantify staining in each of the tumour samples for p16/INK4A abundance. A nuclear and cytoplasmic staining with high Allred score (7-8) was observed in $15(17.1 \%)$ of the MPM cases, a low score (0-3) was observed for $45(51.1 \%)$ and an intermediate score (4-6) was observed for 28 $(31.8 \%)$ cases (Table 1). The median post-diagnosis survival for subjects with high or intermediate Allred score was 25 months; however median post-diagnosis survival for cases with negative score was only 8 months (Figure 2A). The difference in survival between these two groups was significant (log-rank $P<0.001)$. If high $(n=15)$ and intermediate $(n=28)$ expression was separated, their median survival was 29 months and 23 months, respectively (Figure 2B), but this difference was not significant (log-rank $P=0.413)$. The post-diagnosis survival of both the high and the intermediate p16 expression groups were both significantly better than the low expression group (log-rank $P<0.001$ ). Thus sustained p16/INK4A expression even if somewhat reduced is associated with better survival.

Correlation of p16/INK4A expression and chemotherapeutic intervention with survival. Chemotherapeutic intervention was given to 64 patients whereas 24 did not receive chemotherapy. Patients with low or undetectable p16 expression were regarded as p16 negative and those patients that had high or intermediate p16 expression were regarded as p16 positive since the difference in survival between the high and intermediate expression groups was 

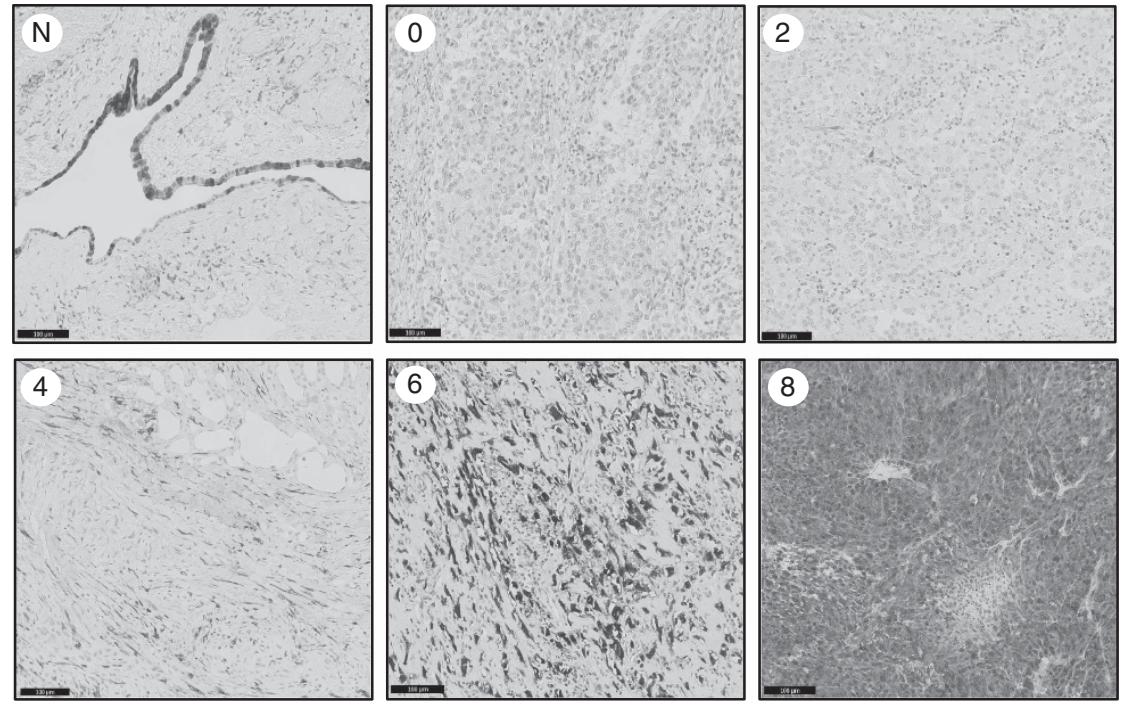

Figure 1. The expression of $\mathrm{p} 16 / \mathrm{INK} 4 \mathrm{~A}$ varies between malignant pleural mesothelioma tumours. Immunohistochemical staining of $\mathrm{p} 16 / \mathrm{INK} 4 \mathrm{~A}$ in normal pleural mesothelium $(\mathrm{N})$ and representative malignant pleural mesothelioma tumour specimens with Allred scores of 0, 2, 4, 6 and 8 .

A

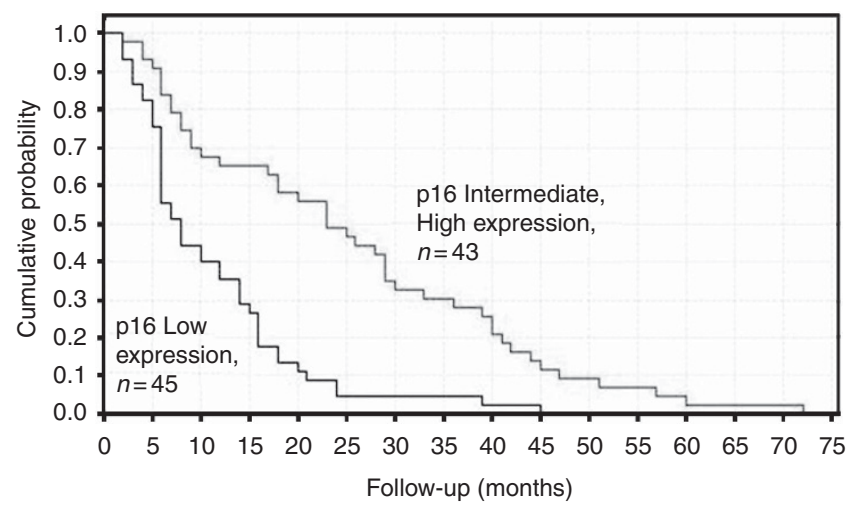

B p16 Expression - all MPM cases

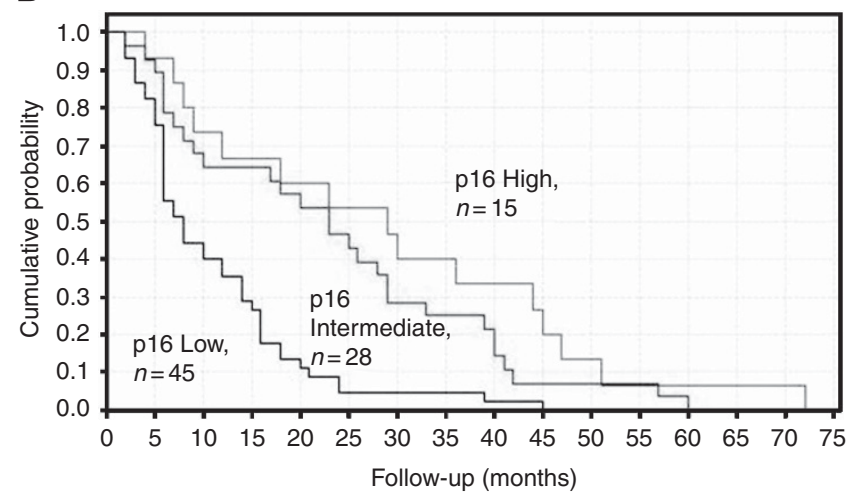

Figure 2. Kaplan-Meier survival analysis of p16/INK4A expression in a cohort of 88 malignant pleural mesothelioma cases. Intermediate and high expression combined ( $n=43)$ vs low expression $(n=45)(A)$. Low expression $(n=45)$ vs high expression $(n=15)$ or intermediate expression only $(n=28)(B)$.

not significant (Figure 2B). Of the patients who did not receive chemotherapy, 15 were p16 negative and 9 were p16 positive. The median post-diagnosis survival for these two groups was 4 and 7 months, respectively (Figure 3), this difference was not significant (log-rank $P=0.069)$. Of the patients who did receive chemotherapy, 30 were p16 negative whereas 34 were p16 positive. The median post-diagnosis survival of these two groups was 14 and 29

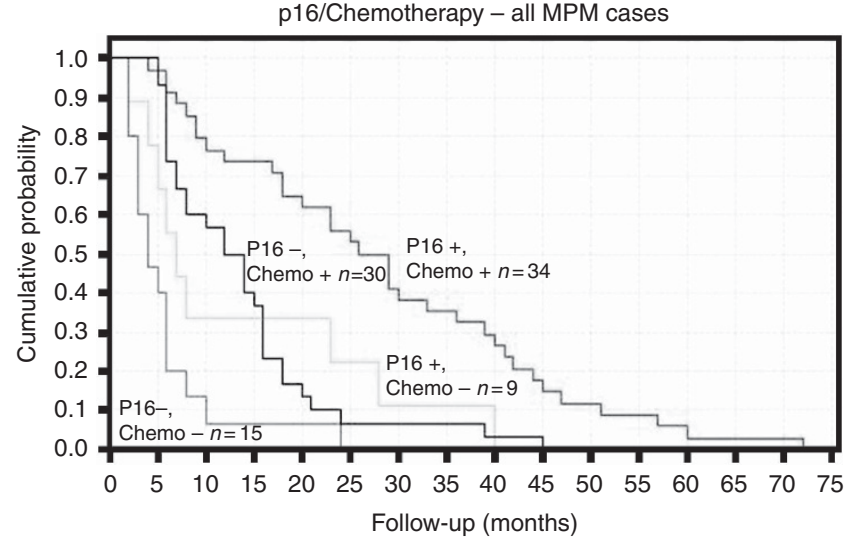

Figure 3. Kaplan-Meier survival analysis of p16/INK4A expression and chemotherapeutic intervention in a cohort of malignant pleural mesothelioma cases. Survival curves for cases that are $\mathrm{p} 16$ positive with chemotherapy $(n=34)$, p16 negative with chemotherapy $(n=30)$, p16 positive without chemotherapy $(n=9)$ and $p 16$ negative without chemotherapy $(n=15)$ are shown.

months, respectively, and was significant (log-rank $P<0.001)$. The sample size for the untreated cases was not large enough to determine significance. The Wilks' Lambda MANOVA statistic was calculated for the effect of p16/INK4A expression and chemotherapeutic treatment on post-diagnosis survival. Both p16/INK4A expression and chemotherapeutic treatment were significant with values of $0.897(\mathrm{~F}=9.32, P=0.03)$ and 0.879 $(\mathrm{F}=11.18, \quad P=0.01)$, respectively. Age at diagnosis was a significant covariate in the Wilks' Lambda analysis with a value of $0.953(\mathrm{~F}=3.99, P=0.049)$, but in this data set histological type and gender were not significant covariates. Sustained p16/INK4A expression by MPM tumours is thus associated with significantly better survival following chemotherapy.

Abundance of p14/ARF and p16/INK4A mRNA in MPM cell lines. T47D breast cancer cell line was used as a positive control for p14 (Figure 4A) and p16 mRNA abundance (Figure 4B). Relative to the T47D breast cancer cell line the normal mesothelial cell line Met-5A expressed p16 mRNA at an abundance of $4.03 \pm 0.39$. The relative p16 abundance for the epithelioid MPM cell lines was $17.27 \pm 2.41$ for MPP-89 cells, $12.19 \pm 0.27$ for MMP 

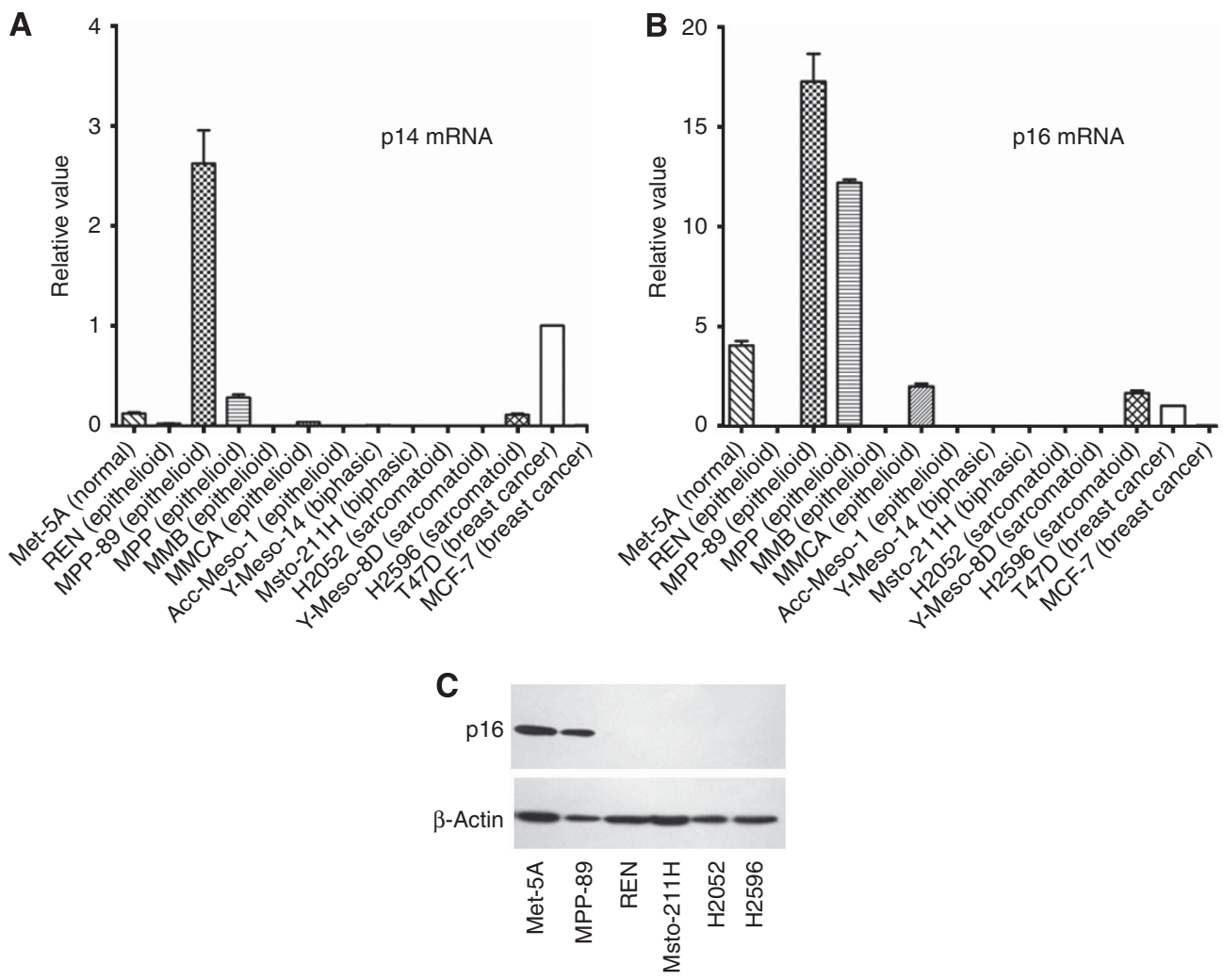

Figure 4. p14/ARF and p16/INK4A expression in malignant pleural mesothelioma cell lines. Quantitative PCR analysis p14/ARF (A) and p16/ INK4A (B) mRNA abundance in 11 MPM cell lines, the Met-5A-transformed pleural mesothelium cell line and two breast carcinoma cell lines (MCF-7 and T47D). Western blot analysis of p16/INK4A expression in the Met-5A-transformed pleural mesothelium cell line and five MPM cell lines (C).

cells, $1.98 \pm 0.24$ for MMCA cells and was not detected $(<0.001$ relative abundance) in the REN, MMB and Acc-Meso-1 epithelioid cells or in the Y-Meso-14 and Msto- $211 \mathrm{H}$ biphasic cell lines. The relative abundance of p16 was $1.64 \pm 0.24$ for the $\mathrm{H} 2956$ sarcomatoid cell line but was not detected in the H2052 or in the Y-Meso-8D sarcomatoid cells. The relative abundance of p14 was $0.12 \pm 0.02$ for the Met-5A cells, $0.02 \pm 0.001$ for the REN cells, $2.62 \pm 0.58$ for the MPP-89 cells, $0.28 \pm 0.05$ for the MMP cells, $0.03 \pm 0.003$ for the MMCA cells, $0.004 \pm 0.0003$ for the Y-Meso-14 and $0.11 \pm 0.02$ for the $\mathrm{H} 2596$ cells but not detected in the MMB, Acc-Meso-1, Msto$211 \mathrm{H}, \mathrm{H} 2052$ or Y-Meso-8D cell lines. These data show a trend for the loss of p16/INK4A mRNA expression in MPM cell lines.

Expression and localisation of p16 in cell lines. Western blot analysis was performed to evaluate p16 protein expression in Met5A, MPP-89, REN, Msto-211H, H2052 and H2956 cells. Met-5A and MPP-89 cells expressed equivalent abundances of p16; however, even on overexposure of the film, p16 protein expression was not detected in the REN, Msto-211H, H2052 and H2956 cell lines (Figure 4C). Confocal imaging of immunofluorescently labelled p16 revealed that it was localised to both the nucleus and cytoplasm, with greater abundance in the cytoplasm of positively staining Met-5A and MPP-89 cells (Figure 5). This subcellular distribution was in agreement with that observed in the patient biopsy samples, where IHC staining was both nuclear and cytoplasmic. H2052 and H2956 cells did not express p16 detectable by Western blotting or immunofluorescence.

\section{DISCUSSION}

Previous studies have found homozygous deletion of p16/INK4A variously in $22-72 \%$ of MPM tumours and $85 \%$ of MPM cell lines (Ichimura et al, 2005; Watkins et al, 2008). Promoter methylation is also a cause of p16/INK4A expression loss in a minority of tumours (Kobayashi et al, 2008; Watkins et al, 2008). We found in this study that p16/INK4A expression was lost (Allred score $\leqslant 3$ ) in $51.1 \%$ of MPM tumours and reduced in a further $31.8 \%$, similar to another study using the same p16/INK4A antibody showing loss of expression ( $<50 \%$ cells staining positive) in $54.5 \%$ of MPM cases (Bahnassy et al, 2012). That study also found a similar frequency of decline in p14 expression amongst MPM cases (50.9\%). The loss of p16/INK4A expression correlated with significantly worse post-diagnosis survival in this cohort of MPM patients. An association between p16/INK4A loss and worse prognosis in MPM has been identified in other studies (Kobayashi et al, 2008) and is regarded as an accurate predictor of 1-year postdiagnosis survival (Lopez-Rios et al, 2006).

The loss of p16 expression is associated with worse responsiveness to multimodal chemotherapy in ovarian carcinoma (Kudoh et al, 2002) and in triple negative breast carcinoma (Arima et al, 2012). Heterologous overexpression of p14/ARF and p16/INK4A in a lung carcinoma cell line enhanced sensitivity to cisplatin (Xie et al, 2005). Sustained p16 expression and chemotherapy responsiveness has not previously been evaluated in MPM. We found that chemotherapeutic intervention was more effective for patients with p16/INK4A expressing tumours. However histological type is also an established predictor of survival and chemotherapeutic efficacy (Flores et al, 2007), and the majority of epithelioid tumours in this study also expressed p16/INK4A (Table 2). Other studies have found that sustained p16/INK4A expression predicts a worse response to chemotherapy in breast carcinoma (Han et al, 2001) and that chemotherapy itself induces p16/INK4A expression as a molecular marker for tissue ageing (Sanoff et al, 2014). The contribution of p16/INK4A to chemotherapeutic efficacy may be complicated by its role in controlling cell cycle progression, so slowing growth and thus attenuating the responsiveness to inhibitors of DNA replication. 


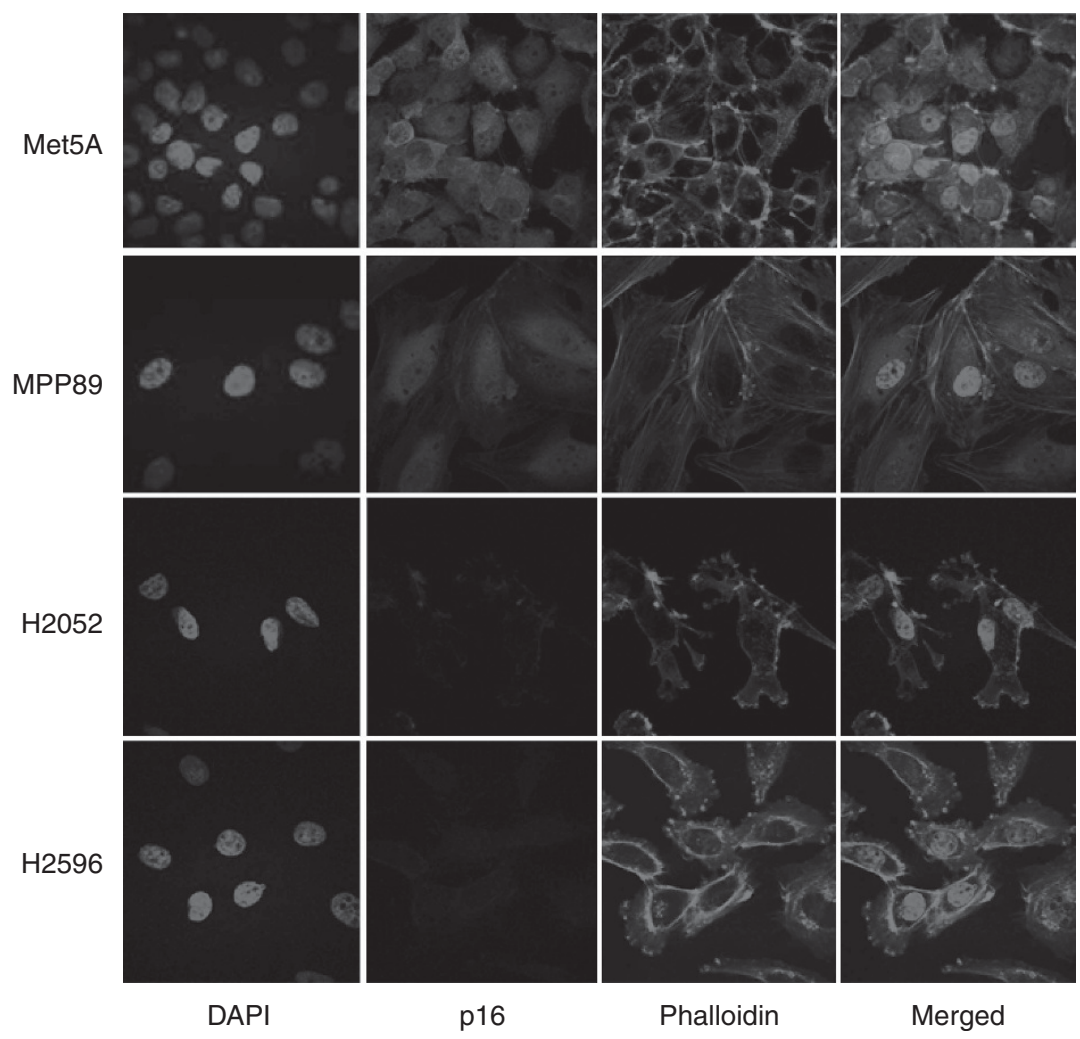

Figure 5. p16/INK4A subcellular distribution in malignant pleural mesothelioma cells. Confocal microscopy showing p16/INK4A subcellular localization and expression in the Met-5A-transformed pleural mesothelium cell line and three MPM cell lines following immunofluorescent staining.

Table 2. Summary of p16 expression distribution between histological types $(n=88)$

\begin{tabular}{|l|c|c|c|}
\hline & $\begin{array}{c}\text { Epithelioid } \\
(\mathbf{n}=\mathbf{7 1})\end{array}$ & $\begin{array}{c}\text { Biphasic } \\
(\mathbf{n}=\mathbf{9})\end{array}$ & $\begin{array}{c}\text { Sarcomatoid } \\
(\mathbf{n}=\mathbf{8})\end{array}$ \\
\hline p16 Expression \\
\hline Negative/low & $33(46.5)$ & $6(66.7)$ & $6(75.0)$ \\
Intermediate & $24(33.8)$ & $2(22.2)$ & $2(25.0)$ \\
High & $14(19.7)$ & $1(11.1)$ & $0(0)$ \\
\hline
\end{tabular}

Values are expressed as $n(\%)$.

Consistent with other studies (Kobayashi et al, 2008; Watkins et al, 2008), we found that expression of p16/INK4A mRNA was detected in only four out of 11 MPM cell lines, for two of the epithelioid cell lines (MPP-89 and MMP) p16/INK4A expression was 10 -fold above that detected in the normal Met-5A mesothelial cells. Expression of p14/ARF was detected in six out of $11 \mathrm{MPM}$ cell lines, but again p14/ARF expression by the MPP-89 and MMP cells was markedly higher than the Met-5A cells. There was some discrepancy between the abundance of p16/INK4A mRNA and the protein abundance detected by western blotting. Recent studies have investigated the post-transcriptional and post-translational regulation of 16/INK4A expression. The p16/INK4A polypeptide has a short half-life in vivo, but the absence of lysine residues precludes degradation by ubiquitin-dependent proteasome activity. It has been proposed that p16/INK4A abundance is in part regulated by REG $\gamma$-dependent activation of the proteasome (Li et al, 2011). There is also an emerging role for microRNA species in the regulation of cell cycle regulatory proteins. The expression of p16/INK4A is suppressed by miR24, a microRNA that is upregulated in many malignancies (Giglio et al, 2013).

When expressed, the majority of the p16/INK4A protein was localised to the cytoplasm with a significant amount of nuclear staining. The distribution of the of p16/INK4A transcription factor to the cytoplasm has been described in other malignancies and is associated with a more invasive phenotype (Nilsson et al, 2004). The differences in subcellular p16/INK4A distribution is due to differential phosphorylation, and the presence/ absence of specific phosphorylated forms of p16/INK4A can be linked to tumour cell phenotype (Nilsson and Landberg, 2006).

Sustained expression of p16/INK4A predicts survival in MPM and correlates with chemotherapeutic efficacy. Post-translational modification of p16/INK4A may be a better predictor of survival and further investigation of $\mathrm{p} 16 / \mathrm{INK} 4 \mathrm{~A}$ phosphorylation in MPM is needed.

\section{ACKNOWLEDGEMENTS}

This work was funded by a $\mathrm{PhD}$ scholarship from the Health Research Board (Ireland) to CJ and by a Science Foundation Ireland award (12/TIDA/I2372) to WT.

\section{REFERENCES}

Allred DC, Clark GM, Elledge R, Fuqua SA, Brown RW, Chamness GC, Osborne CK, McGuire WL (1993) Association of p53 protein expression with tumor cell proliferation rate and clinical outcome in node-negative breast cancer. J Natl Cancer Inst 85(3): 200-206.

Arima Y, Hayashi N, Hayashi H, Sasaki M, Kai K, Sugihara E, Abe E, Yoshida A, Mikami S, Nakamura S, Saya H (2012) Loss of p16 expression is associated with the stem cell characteristics of surface markers and therapeutic resistance in estrogen receptor-negative breast cancer. Int J Cancer 130(11): 2568-2579.

Bahnassy AA, Zekri AR, Abou-Bakr AA, El-Deftar MM, El-Bastawisy A, Sakr MA, El-Sherif GM, Gaafar RM (2012) Aberrant expression of cell cycle regulatory genes predicts overall and disease free survival in 
malignant pleural mesothelioma patients. Exp Mol Pathol 93(1): 154-161.

Bianchi C, Bianchi T (2007) Malignant mesothelioma: global incidence and relationship with asbestos. Ind Health 45(3): 379-387.

Burke JR, Liban TJ, Restrepo T, Lee HW, Rubin SM (2014) Multiple mechanisms for E2F binding inhibition by phosphorylation of the retinoblastoma protein C-terminal domain. J Mol Biol 426(1): 245-255.

Cacciotti P, Strizzi L, Vianale G, Iaccheri L, Libener R, Porta C, Tognon M, Gaudino G, Mutti L (2002) The presence of simian-virus 40 sequences in mesothelioma and mesothelial cells is associated with high levels of vascular endothelial growth factor. Am J Respir Cell Mol Biol 26(2): 189-193.

Dacic S, Kothmaier H, Land S, Shuai Y, Halbwedl I, Morbini P, Murer B, Comin C, Galateau-Salle F, Demirag F, Zeren H, Attanoos R, Gibbs A, Cagle P, Popper H (2008) Prognostic significance of p16/cdkn2a loss in pleural malignant mesotheliomas. Virchows Arch 453(6): 627-635.

Flores RM, Zakowski M, Venkatraman E, Krug L, Rosenzweig K, Dycoco J, Lee C, Yeoh C, Bains M, Rusch V (2007) Prognostic factors in the treatment of malignant pleural mesothelioma at a large tertiary referral center. J Thorac Oncol 2(10): 957-965.

Giglio S, Cirombella R, Amodeo R, Portaro L, Lavra L, Vecchione A (2013) MicroRNA miR-24 promotes cell proliferation by targeting the CDKs inhibitors p27Kip1 and p16INK4a. J Cell Physiol 228(10): 2015-2023.

Han S, Ahn SH, Park K, Bae BN, Kim KH, Kim HJ, Kim YD, Kim HY (2001) P16INK4a protein expression is associated with poor survival of the breast cancer patients after CMF chemotherapy. Breast Cancer Res Treat 70(3): 205-212.

Harrington DP, Fleming TR (1982) A class of rank procedures for censored survival data. Biometrika 69: 553-566.

Ichimura T, Yamamura H, Sasamoto K, Tominaga Y, Taoka M, Kakiuchi K, Shinkawa T, Takahashi N, Shimada S, Isobe T (2005) 14-3-3 proteins modulate the expression of epithelial $\mathrm{Na}+$ channels by phosphorylationdependent interaction with Nedd4-2 ubiquitin ligase. J Biol Chem 280(13): 13187-13194.

Jennings CJ, O'Grady A, Cummins R, Murer B, Al-Alawi M, Madden SF, Mutti L, Harvey BJ, Thomas W, Kay EW (2012) Sustained expression of steroid receptor coactivator SRC-2/TIF-2 is associated with better prognosis in malignant pleural mesothelioma. J Thorac Oncol 7(1): 243-248.

Jennings CJ, Walsh PM, Deady S, Harvey BJ, Thomas W (2014) Malignant pleural mesothelioma incidence and survival in the Republic of Ireland 1994-2009. Cancer Epidemiol 38(1): 35-41.

Kanellou P, Zaravinos A, Zioga M, Stratigos A, Baritaki S, Soufla G, Zoras O, Spandidos DA (2008) Genomic instability, mutations and expression analysis of the tumour suppressor genes p14(ARF), p15(INK4b), p16(INK4a) and p53 in actinic keratosis. Cancer Lett 264(1): 145-161.

Ke Y, Reddel RR, Gerwin BI, Reddel HK, Somers AN, McMenamin MG, LaVeck MA, Stahel RA, Lechner JF, Harris CC (1989) Establishment of a human in vitro mesothelial cell model system for investigating mechanisms of asbestos-induced mesothelioma. Am J Pathol 134(5): 979-991.

Kobayashi N, Toyooka S, Yanai H, Soh J, Fujimoto N, Yamamoto H, Ichihara S, Kimura K, Ichimura K, Sano Y, Kishimoto T, Date H (2008) Frequent p16 inactivation by homozygous deletion or methylation is associated with a poor prognosis in Japanese patients with pleural mesothelioma. Lung Cancer 62(1): 120-125.

Kudoh K, Ichikawa Y, Yoshida S, Hirai M, Kikuchi Y, Nagata I, Miwa M, Uchida K (2002) Inactivation of p16/CDKN2 and p15/MTS2 is associated with prognosis and response to chemotherapy in ovarian cancer. Int J Cancer 99(4): 579-582.

Le Stang N, Belot A, Gilg Soit Ilg A, Rolland P, Astoul P, Bara S, Brochard P, Danzon A, Delafosse P, Grosclaude P, Guizard AV, Imbernon E, Lapotre-Ledoux B, Ligier K, Molinie F, Pairon JC, Sauleau EA, Tretarre B, Velten M, Bossard N, Goldberg M, Launoy G, Galateau-Salle F (2010) Evolution of pleural cancers and malignant pleural mesothelioma incidence in France between 1980 and 2005. Int J Cancer 126(1): 232-238.
Li J, Poi MJ, Tsai MD (2011) Regulatory mechanisms of tumor suppressor P16(INK4A) and their relevance to cancer. Biochemistry 50(25): 5566-5582.

Li YL, Ye F, Hu Y, Lu WG, Xie X (2009) Identification of suitable reference genes for gene expression studies of human serous ovarian cancer by real-time polymerase chain reaction. Anal Biochem 394(1): 110-116.

Lopez-Rios F, Chuai S, Flores R, Shimizu S, Ohno T, Wakahara K, Illei PB, Hussain S, Krug L, Zakowski MF, Rusch V, Olshen AB, Ladanyi M (2006) Global gene expression profiling of pleural mesotheliomas: overexpression of aurora kinases and P16/CDKN2A deletion as prognostic factors and critical evaluation of microarray-based prognostic prediction. Cancer Res 66(6): 2970-2979.

Nilsson K, Landberg G (2006) Subcellular localization, modification and protein complex formation of the cdk-inhibitor p16 in Rb-functional and Rb-inactivated tumor cells. Int J Cancer 118(5): 1120-1125.

Nilsson K, Svensson S, Landberg G (2004) Retinoblastoma protein function and p16INK4a expression in actinic keratosis, squamous cell carcinoma in situ and invasive squamous cell carcinoma of the skin and links between p16INK4a expression and infiltrative behavior. Mod Pathol 17(12): 1464-1474.

Orengo AM, Spoletini L, Procopio A, Favoni RE, De Cupis A, Ardizzoni A, Castagneto B, Ribotta M, Betta PG, Ferrini S, Mutti L (1999) Establishment of four new mesothelioma cell lines: characterization by ultrastructural and immunophenotypic analysis. Eur Respir J 13(3): $527-534$.

Sanoff HK, Deal AM, Krishnamurthy J, Torrice C, Dillon P, Sorrentino J, Ibrahim JG, Jolly TA, Williams G, Carey LA, Drobish A, Gordon BB, Alston S, Hurria A, Kleinhans K, Rudolph KL, Sharpless NE, Muss HB (2014) Effect of cytotoxic chemotherapy on markers of molecular age in patients with breast cancer. J Natl Cancer Inst 106(4): dju057.

Sherr CJ (1996) Cancer cell cycles. Science 274(5293): 1672-1677.

Smythe WR, Kaiser LR, Hwang HC, Amin KM, Pilewski JM, Eck SJ, Wilson JM, Albelda SM (1994) Successful adenovirus-mediated gene transfer in an in vivo model of human malignant mesothelioma. Ann Thorac Surg 57(6): 1395-1401.

Spugnini EP, Campioni M, D’Avino A, Caruso G, Citro G, Baldi A (2007) Cell-cycle molecules in mesothelioma: an overview. J Exp Clin Cancer Res 26(4): 443-449.

Steele JP (2005) Prognostic factors for mesothelioma. Hematol Oncol Clin North Am 19(6): 1041-1052.

Sterman DH, Albelda SM (2005) Advances in the diagnosis, evaluation, and management of malignant pleural mesothelioma. Respirology 10(3): 266-283.

Usami N, Fukui T, Kondo M, Taniguchi T, Yokoyama T, Mori S, Yokoi K, Horio Y, Shimokata K, Sekido Y, Hida T (2006) Establishment and characterization of four malignant pleural mesothelioma cell lines from Japanese patients. Cancer Sci 97(5): 387-394.

Watkins JL, Lewandowski KT, Meek SE, Storz P, Toker A, Piwnica-Worms H (2008) Phosphorylation of the Par-1 polarity kinase by protein kinase D regulates 14-3-3 binding and membrane association. Proc Natl Acad Sci U S A 105(47): 18378-18383.

Wong L, Zhou J, Anderson D, Kratzke RA (2002) Inactivation of p16INK4a expression in malignant mesothelioma by methylation. Lung Cancer 38(2): 131-136.

Xie QC, Hu YD, Wang LL, Chen ZT, Diao XW, Wang ZX, Guan HJ, Zhu B, Sun JG, Duan YZ, Chen FL, Nian WQ (2005) The co-transfection of p16(INK4a) and p14(ARF) genes into human lung cancer cell line A549 and the effects on cell growth and chemosensitivity. Colloids Surf B Biointerfaces 46(3): 188-196.

This work is published under the standard license to publish agreement. After 12 months the work will become freely available and the license terms will switch to a Creative Commons AttributionNonCommercial-Share Alike 4.0 Unported License

Supplementary Information accompanies this paper on British Journal of Cancer website (http://www.nature.com/bjc) 